\title{
OMP-BASED ALGORITHM FOR MINERAL REFLECTANCE SPECTRA DECONVOLUTION FROM HYPERSPECTRAL IMAGES
}

\author{
Ronan Rialland $^{1, *}$, Charles Soussen ${ }^{2}$, Rodolphe Marion ${ }^{1}$, Véronique Carrère ${ }^{3}$ \\ ${ }^{1} \mathrm{CEA} / \mathrm{DAM} / \mathrm{DIF}$, Arpajon, France \\ ${ }^{2}$ L2S, CentraleSupélec - CNRS - Université Paris-Saclay, Gif-sur-Yvette, France \\ ${ }^{3}$ UMR-CNRS 6112 - LPG Nantes, Université de Nantes, Nantes, France
}

\begin{abstract}
The absorption positions and shapes are key information to identify and characterize a mineral from its reflectance spectrum. With the development of new airborne and satellite-borne hyperspectral sensors, automatic methods have to be developed to extract and analyze this useful information. A flexible deconvolution procedure, able to deal with various sensor characteristics and a wide variety of minerals of interest, is proposed. The approach is based on the sparse representation of the spectrum and the use of a greedy algorithm, the Non-Negative Orthogonal Matching Pursuit algorithm. First, NNOMP is adapted to deal with a parameteric physical model of mineral reflectance spectra. Then, noise statistical information is taken into account to improve the detection of small absorptions while minimizing overfitting effects. The procedure is tested on real data from two quarries in France. Results show the potential of our procedure for the estimation of a consistent number of absorptions whose parameters can be used to analyze the mineralogy.
\end{abstract}

Index Terms - Mineral reflectance spectra, Deconvolution procedure, Hyperspectral image, Orthogonal Matching Pursuit, EGO model.

\section{INTRODUCTION}

Hyperspectral imagery in the solar reflective domain (VNIR, (Visible Near-InfraRead) [400 - 1300] $\mathrm{nm}$ and SWIR (ShortWave InfraRed) $[1300-2500] \mathrm{nm}$ ) provides a reflectance spectrum for each pixel of an image allowing to retrieve and map physico-chemical properties of the observed surface. In particular, spectra of minerals are composed of specific, or diagnostic, absorption bands, the position of which depends mainly on the chemical composition [1]. Absorptions in the SWIR are generally narrow and deep, whereas in the VNIR they tend to be broader and weaker because they correspond to various physical phenomena (rotation, vibration, stretching and bending of molecule bonds in the SWIR, crystal field, electronic transitions in the VNIR). The overall shape, also

\footnotetext{
*Corresponding author email: ronan.rialland@cea.fr
}

called continuum, changes with surface conditions (e.g., grain size, humidity), so that a simple comparison of a spectrum with a huge database using a metric generally fails.

Thanks to the development of new airborne and satelliteborne sensors, hyperspectral images are increasingly acquired over large areas of mineralogical interest so that detailed spectral analysis can be performed. The development of automatic algorithms able to deal with various sensor characteristics and a wide variety of minerals of interest is an important objective which still remains challenging. Among the various methods available in the literature (e.g., spectral indices [2] and references therein, Tetracorder [3]), spectral deconvolution is a method of choice because the full spectrum is taken into account, and it relies as much as possible on a physically-based reflectance model.

In this paper, spectral deconvolution is combined with the EGO model (Exponential Gaussian Optimization) [4]. The EGO model is derived from the MGM model (Modified Gaussian Model) [5] which is well-suited for iron related features in the VNIR but not in the SWIR where absorptions are generally narrower and often asymmetrical. This specificity is taken into account by the introduction of an asymmetry factor in (2). One may note that the authors have also introduced a parameter to take into account the saturation of the absorption bands [6], which is not considered here for sake of simplicity. Thus, the deconvolution procedure aims at estimating the positions $\mu$ and shape parameters (widths $\sigma$, amplitudes $s$, asymmetry parameters $k$ ) of the $N$ modified Gaussians that best fit the absorption bands of the logarithm of the reflectance. The continuum is here removed beforehand using a convex hull approach [7]. The model of the reflectance spectrum $\rho^{C R}$ with continuum removed reads

$$
\ln \rho^{C R}(\lambda, \theta)=\sum_{i=1}^{N} G_{i}\left(\lambda, \theta_{G_{i}}\right)
$$

where the modified Gaussians are defined by

$$
G_{i}\left(\lambda, \theta_{G_{i}}\right)=s_{i} \exp \left(-\frac{1}{2} \frac{\left(\lambda-\mu_{i}\right)^{2}}{\left(\sigma_{i}-k_{i}\left(\lambda-\mu_{i}\right)\right)^{2}}\right)
$$


with $\theta_{G_{i}}=\left\{s_{i}, \mu_{i}, \sigma_{i}, k_{i}\right\} . \theta=\left\{N, \theta_{G_{1}}, \ldots, \theta_{G_{N}}\right\}$ gathers the full set of parameters.

Several solutions have been proposed to estimate the model parameters $\theta_{G_{i}}$ and select the order $N$ of the model automatically. They generally implement two steps: an initial estimation of the parameters followed by an optimization (nonlinear least-squares) algorithm. This approach was adopted in the AGM procedure (Automatized Gaussian Model) [8], [9] previously developed by the authors. We focus here on the initialization step. One can use spectral derivatives to estimate the number of Gaussians and their positions [8], which is noise-sensitive and may lead to overfitting. Transformation methods such as the Continuous Wavelet Transform [10] are not satisfactory because they require to set many parameters which cannot be adjusted to values adapted to both the VNIR and the SWIR due the great diversity of spectral signatures of minerals.

In this paper, to initialize the parameters, an orthogonal matching pursuit based algorithm with non-negativity constraints, the NNOMP (Non-Negative Orthogonal Matching Pursuit) algorithm [11], is used. Since the principle of this approach is to approximate a signal as a linear combination of a limited number of atoms, it is well-suited to fit the absorption bands in the reflectance spectrum of a mineral with the EGO model. Here, NNOMP is adapted to take into account the spectrally varying noise in the image to improve the detection of small amplitude absorptions while minimizing overfitting effects.

Hereafter, the NNOMP algorithm for deconvolution of mineral reflectance spectra is first described. Then, its extension to take noise statistics into account is explained. Finally, experimental results on real spectra acquired on two quarries in France are presented. Further validation and comparisons to ground truths will be presented during the conference.

\section{METHODS}

\subsection{NNOMP algorithm for mineral reflectance spectra deconvolution}

NNOMP is a sparse approximation algorithm under nonnegativity constraints. It can be interpreted as an optimization algorithm dedicated to the optimization problem (3), where $\mathbf{a} \in \mathbb{R}^{N_{\lambda}}$ represents an input signal (e.g., a spectrum related to a given pixel) and $\mathbf{H} \in \mathbb{R}^{N_{\lambda} \times N_{\text {atom }}}$ is a dictionary matrix including all the possible spectral features embedded in the data signal a. The problem is formulated as

$$
\min _{\mathbf{s}}\|\mathbf{a}-\mathbf{H s}\|_{2}^{2} \quad \text { s.t. } \quad\left\{\begin{array}{l}
\mathbf{s} \geq \mathbf{0} \\
\|\mathbf{s}\|_{0} \leq N_{\max }
\end{array}\right.
$$

where the $\ell_{0}$ norm $\|\mathbf{s}\|_{0}$ counts the number of non-zero entries in vector $\mathbf{s}$.

The principle of NNOMP (see Algorithm 11) is to gradually select atoms in $\mathbf{H}$ in order to refine the approxima-

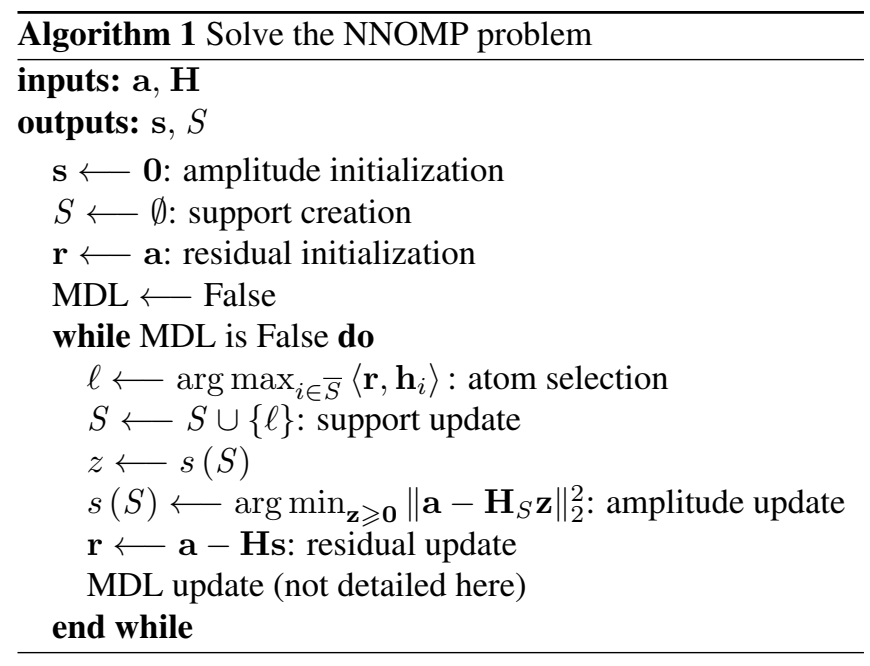

tion $\mathbf{a} \approx$ Hs. First, $\mathbf{s} \in \mathbb{R}^{N_{\text {atom }}}$ is initialized to $\mathbf{0}$ and the support of $\mathbf{s}$ is thus set to $S=\emptyset$. Then, at each iteration, NNOMP proceeds as follows. A new atom $\mathbf{h}_{\ell}$ (i.e., a column) of $\mathbf{H}$ is selected and added to the support $S$. Then, the selected amplitudes $\mathbf{z}$ are adjusted, using a NNLS (NonNegative Least-Squares) algorithm, to fit the absorption part of the spectrum a with the selected atoms $\mathbf{H}_{S}$. Finally, the approximation residual is updated. NNOMP yields as output the selected atoms of $\mathbf{H}$ and their respective non-negative amplitudes. Three alternative stopping criteria can be used: (i) a maximum number $N_{\max }$ of atoms is imposed; (ii) the square norm of the residual $\mathbf{r}$ is less than a threshold; or (iii) a criterion related to the Minimum Description Length (MDL) principle [12] is used. This is the stopping criterion used in this study.

To deal with mineral reflectance spectra, a specific shape for the atoms in $\mathbf{H}$ is proposed. Thus, each atom is an EGO modified Gaussian with a specific width-asymmetry couple at a given location in the VNIR and SWIR domains. The choice of a large number of width-asymmetry couples ensures the presence of mineral diagnostic absorptions in the dictionary. Moreover, in order to apply the existing NNOMP implementation in [11], the columns of $\mathbf{H}$ are normalized and $\mathbf{s}$ corresponds to the amplitudes of the EGO modified Gaussians.

Thanks to the creation of a specific dictionary and the use of adapted stopping criteria, NNOMP gives the possibility to analyze a large variety of minerals with a consistent number of absorptions of varying shapes.

\subsection{NNOMP including noise statistics}

In order to work with various sensor characteristics and improve the deconvolution procedure, noise statistical information is used in NNOMP. Noise is assumed to be zero-mean, Gaussian and independent from one spectral band to another. However, the noise is not identically distributed since the noise variance $\sigma_{\lambda}^{2}$ is known to vary with respect to the spectral band $\lambda$. The noise covariance matrix $\mathbf{W}=\operatorname{diag}\left(\sigma_{\lambda}^{2}\right)$ is 
thus diagonal (but differs from the identity matrix) and can be either calculated knowing the sensor characteristics, or estimated [13]. The minimization problem can then be expressed with non-negative and sparsity constraints as

$$
\min _{\mathbf{s}}\|\boldsymbol{\Gamma}(\mathbf{a}-\mathbf{H} \mathbf{s})\|_{2}^{2}=\min _{\widetilde{\mathbf{s}}}\left\|\mathbf{\Gamma} \mathbf{a}-\mathbf{H}_{1}\left(\Delta^{-1} \widetilde{\mathbf{s}}\right)\right\|_{2}^{2}
$$

where $\boldsymbol{\Gamma}=\mathbf{W}^{-1 / 2}$. The reparametrization $\mathbf{H}_{1}=\boldsymbol{\Gamma} \mathbf{H} \Delta$ and $\mathbf{s}=\Delta^{-1} \widetilde{\mathbf{s}}$ is done to ensure the normalization of the columns of $\mathbf{H}$ with a diagonal matrix $\Delta$. As the noise variance depends on wavelength, NNOMP is more suited to work with different sensors and is able to discriminate weak absorptions.

\section{DATA SET}

To validate the proposed method, hyperspectral images were acquired over two quarries in France, Fig. 1, with HySpex cameras (https://www.hyspex.com). Three minerals of interest, with different spectral signatures, can be observed: gypsum, carbonates (Cherves-Richemont) and kaolinite (Chevanceaux). They present different physical and chemical characteristics and are used in the production of plasterboard or as aggregates (Cherves-Richemont) and refractory ceramics (Chevanceaux).

Images were acquired in September 2019, with a $0.5 \mathrm{~m}$ and a $1.0 \mathrm{~m}$ spatial resolution for the VNIR and SWIR respectively. The numbers of bands were respectively 160 and 162 for the VNIR and SWIR with $\mathrm{a} \approx 4 \mathrm{~nm}$ and $\approx 7 \mathrm{~nm}$ spectral resolution. In this study, only SWIR images are used. Images were atmospherically corrected using ATCOR4 [14]. To improve the signal-to-noise ratio, reflectance images were spatially downsampled to $5 \mathrm{~m}$. Two spectral masks were applied around $1400 \mathrm{~nm}$ and $1900 \mathrm{~nm}$ to avoid effects of atmospheric water vapor absorptions.

Samples were collected after the image acquisition in order to create a spectral database gathering the various spectral

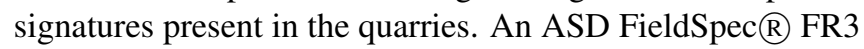
was used for laboratory measurements.

\section{EXPERIMENTAL RESULTS: DECONVOLUTION PROCEDURE INCLUDING NOISE STATISTICS}

The validation of the proposed deconvolution procedure is carried out on a kaolinite spectrum from the hyperspectral image of Chevanceaux. The pixel is chosen on a pile of kaolinite. To avoid spectral mask effects due to atmospheric water vapor absorptions, only wavelengths between $2100 \mathrm{~nm}$ and $2400 \mathrm{~nm}$ are kept. The noise covariance $\mathbf{W}$ is estimated using HYSIME [13] and the continuum is removed [7]. The dictionary $\mathbf{H}$ formed of EGO Gaussians is created with a large number of widths $\sigma \in\left[1, \frac{N_{\lambda}}{4}\right]$ and asymmetry factors $k \in\left[-\frac{1}{2}, \frac{1}{2}\right]$ yielding 129800 atoms. To estimate adaptively the number of Gaussians, the MDL stopping criterion (dic-

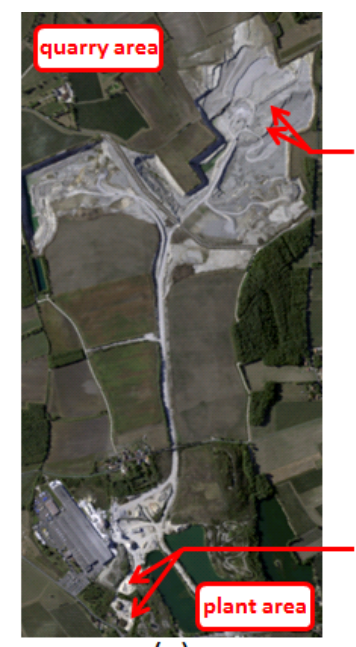

(a)

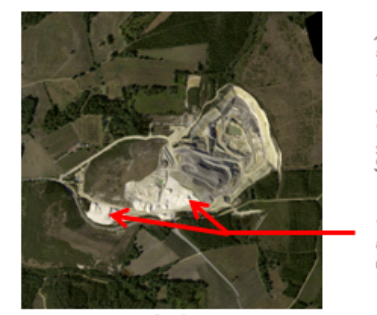

(b)

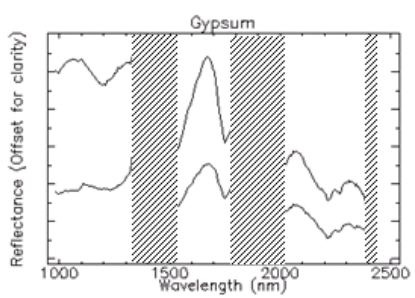

(c)

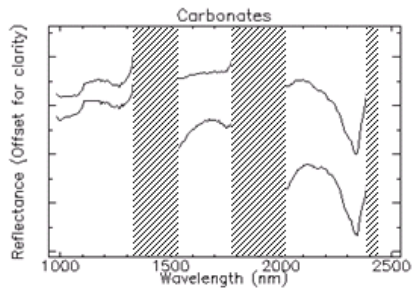

(d)
Fig. 1. Hyperspectral images acquired over (a) ChervesRichemont and (b) Chevanceaux quarries. (c) Gypsum spectra on the quarry layer. (d) Carbonates spectra from piles used as aggregates. (e) Kaolinite spectra with different concentrations of alumina (note the absorption doublet varying in shape depending on the pile).

tionary $\mathbf{H}$ ) which can be adapted to the case of known noise statistics (dictionary $\mathbf{H}_{1}$ ) is used in NNOMP.

A kaolinite spectrum is defined by its absorption doublet at $2160 \mathrm{~nm}$ and $2210 \mathrm{~nm}$. These two absorptions have similar shapes. The spectrum has also secondary absorptions at $2310 \mathrm{~nm}, 2350 \mathrm{~nm}$ and $2380 \mathrm{~nm}$, often weak and difficult to separate from the noise.

Results of the deconvolution procedure are presented in Fig. 2. NNOMP, applied to dictionary $\mathbf{H}$, fits the spectrum with only three EGO Gaussians, two for the global shape of the absorption bands and one for the absorption at $2210 \mathrm{~nm}$. NNOMP, applied to dictionary $\mathbf{H}_{1}$, shows a better fit. The absorption doublet is fitted with three EGO Gaussians, one for the global shape, broad and centered at $2187 \mathrm{~nm}$, and two centered at $2157 \mathrm{~nm}$ and $2205 \mathrm{~nm}$ for the absorptions. Note that the widths and the amplitudes fit to our expectations. Moreover, two small absorptions are also identified at 2314 $\mathrm{nm}$ and $2398 \mathrm{~nm}$ and a broad one fits the drop off towards longer wavelengths.

More EGO Gaussians are selected with the proposed deconvolution procedure but are coherent with a physical interpretation. Even if the first selected atoms corresponds to 
the global shape of the absorption bands, taking into account noise statistical information improves the detection of small absorptions and doublets. Overfitting is limited thanks to the sparsity constraint and EGO parameters can be used for mineralogical identification and characterization.
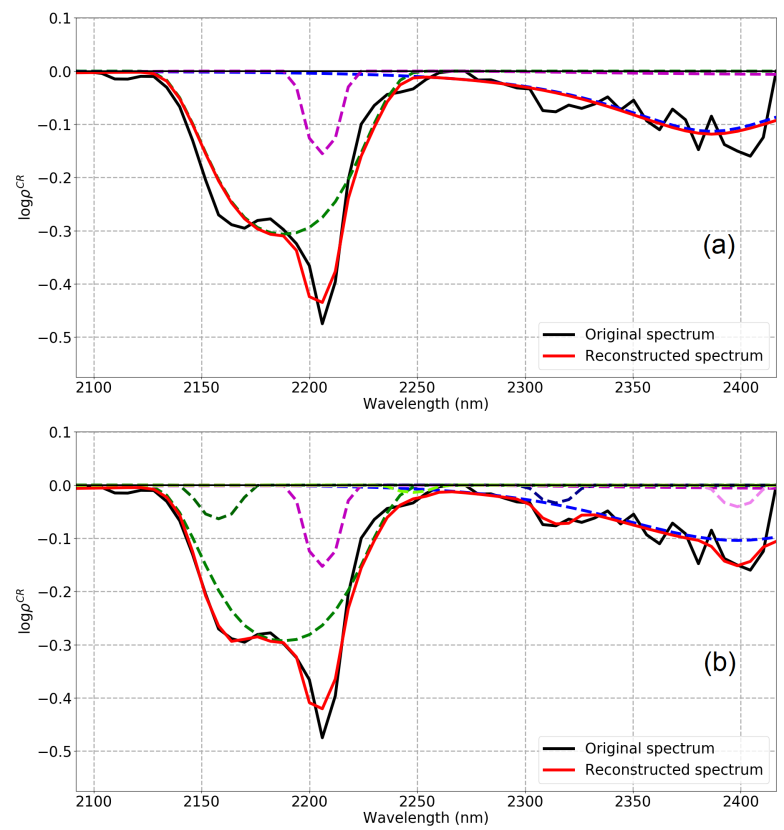

Fig. 2. Deconvolution of a kaolinite spectrum (black), between $2100 \mathrm{~nm}$ and $2400 \mathrm{~nm}$, after continuum removal. a) Reconstructed spectrum (red) with NNOMP, applied to dictionary $\mathbf{H}$ (unknown noise statistics). (b) Reconstructed spectrum (red) with NNOMP, applied to dictionary $\mathbf{H}_{1}$ (known noise statistics). Selected atoms of $\mathbf{H}$ and $\mathbf{H}_{1}$ are also represented for both reconstructions in dotted lines.

\section{CONCLUSION}

In this paper, an existing OMP-based approach is adapted to mineral reflectance spectra and noise statistical information is included in the deconvolution procedure. It gives the possibility to $(i)$ find absorptions with varying shapes, even close to noise, and (ii) use the estimated parameters in an identification and characterization procedure of the mineral and its substrate. Several improvements are considered to the proposed method as for example the use of the complete EGO model with a saturation parameter or the development of a procedure to remove possible artifacts due to the presence of spectral masks. The proposed deconvolution procedure is used in AGM to initialize the absorption part of the spectrum and a complete validation will be presented during the conference.

\section{ACKNOWLEGMENTS}

The authors acknowledge the LPG Nantes and GEOFIT EXPERT for the acquisition of the HySpex images. Imerys and Garandeau firms are thanked for their collaboration.

\section{REFERENCES}

[1] R. N. Clark, Chapter 1: Spectroscopy of Rocks and Minerals, and Principles of Spectroscopy, in Manual of Remote Sensing, Volume 3, Remote Sensing for the Earth Sciences, John Wiley and Sons, New York, 1999.

[2] S. Asadzadeh and C. R. de Souza Filho, "A review on spectral processing methods for geological remote sensing," International Journal of Applied Earth Observation and Geoinformation, vol. 47, pp. 69 - 90, 2016.

[3] R. N. Clark, G. A. Swayze, K. E. Livo, R. F. Kokaly, S. J. Sutley, J. B. Dalton, R. R. McDougal Robert R., and C. A. Gent, "Imaging spectroscopy: Earth and planetary remote sensing with the usgs tetracorder and expert systems," Journal of Geophysical Research: Planets, vol. 108, no. E12, pp. 5131, 2003.

[4] L. Pompilio, G. Pedrazzi, M. Sgavetti, E. A. Cloutis, M. A. Craig, and T. L. Roush, "Exponential gaussian approach for spectral modeling: The EGO algorithm I. band saturation," Icarus, vol. 201, no. 2, pp. 781 - 794, 2009.

[5] J. M. Sunshine, C. M. Pieters, and S. F. Pratt, "Deconvolution of mineral absorption bands: An improved approach," Journal of Geophysical Research: Solid Earth, vol. 95, no. B5, pp. 6955-6966, 1990.

[6] L. Pompilio, G. Pedrazzi, E. A. Cloutis, M. A. Craig, and T. L. Roush, "Exponential gaussian approach for spectral modelling: The EGO algorithm II. band asymmetry," Icarus, vol. 208, no. 2, pp. $811-823,2010$.

[7] R. N. Clark and T. L. Roush, "Reflectance spectroscopy: Quantitative analysis techniques for remote sensing applications," Journal of Geophysical Research: Solid Earth, vol. 89, no. B7, pp. 6329-6340, 1984.

[8] M. Brossard, R. Marion, and V. Carrère, "Deconvolution of SWIR reflectance spectra for automatic mineral identification in hyperspectral imaging," Remote Sensing Letters, vol. 7, no. 6, pp. 581-590, 2016.

[9] R. Marion and V. Carrère, "Mineral mapping using the Automatized Gaussian Model (AGM) - application to two industrial french sites at gardanne and thann," Remote Sensing, vol. 10, no. 1, 2018.

[10] P. Du, W. A. Kibbe, and S. M. Lin, "Improved peak detection in mass spectrum by incorporating continuous wavelet transformbased pattern matching," Bioinformatics, vol. 22, no. 17, pp. 2059-2065, 072006.

[11] T. T. Nguyen, J. Idier, C. Soussen, and E. Djermoune, "Nonnegative orthogonal greedy algorithms," IEEE Transactions on Signal Processing, vol. 67, no. 21, pp. 5643-5658, Nov 2019.

[12] F. De Ridder, R. Pintelon, J. Schoukens, and D. P. Gillikin, "Modified AIC and MDL model selection criteria for short data records," IEEE Transactions on Instrumentation and Measurement, vol. 54, no. 1, pp. 144-150, Feb 2005.

[13] J. M. Bioucas-Dias and J. M. P. Nascimento, "Hyperspectral subspace identification," IEEE Transactions on Geoscience and Remote Sensing, vol. 46, no. 8, pp. 2435-2445, Aug 2008.

[14] R. Richter and D. Schlapfer, "Atmospheric/topographic correction for airborne imagery: Atcor-4 user guide," DLR IB, pp. 565-02, 2012. 